\title{
Vacuum Contributions in a Chiral Effective Lagrangian for Nuclei
}

\author{
R. J. Furnstahl and Hua-Bin Tang \\ Department of Physics \\ The Ohio State University, Columbus, Ohio 43210 \\ Brian D. Serot \\ Physics Department and Nuclear Theory Center \\ Indiana University, Bloomington, Indiana 47405
}

(January 25, 1995)

\begin{abstract}
A relativistic hadronic model for nuclear matter and finite nuclei, which incorporates nonlinear chiral symmetry and broken scale invariance, is presented and applied at the one-baryon-loop level to finite nuclei. The model contains an effective light scalar field that is responsible for the mid-range nucleon-nucleon attraction and which has anomalous scaling behavior. Oneloop vacuum contributions in this background scalar field at finite density are constrained by low-energy theorems that reflect the broken scale invariance of quantum chromodynamics. A mean-field energy functional for nuclear matter and nuclei is derived that contains small powers of the fields and their derivatives, and the validity of this truncation is discussed. Good fits to the bulk properties of finite nuclei and single-particle spectra are obtained.
\end{abstract}

\section{INTRODUCTION}

Descriptions of nuclear matter and finite nuclei, which are ultimately governed by the physics of low-energy quantum chromodynamics (QCD), are efficiently formulated using lowenergy degrees of freedom - the hadrons. In the absence of direct derivations from QCD, such effective descriptions should be constrained by the underlying symmetries of QCD, both broken and unbroken. Nevertheless, the appropriate realization of these symmetries for phenomenological models is not yet established. In this paper, we explore some consequences of applying QCD symmetry constraints to a relativistic model of finite nuclei that features a light scalar meson.

At present, the most developed framework for constraining hadronic physics by QCD symmetries is chiral perturbation theory (ChPT) [1], which provides a systematic expansion 
in energy for low-energy scattering processes. The degrees of freedom are the Goldstone bosons (pions, etc.) and, when appropriate, nucleons. This approach builds in constraints due to chiral symmetry without any additional constraints on the dynamics or ad hoc model assumptions; physics beyond chiral symmetry is incorporated through constants in the lowenergy lagrangian, which are usually determined from experiment. Because additional constants are needed at each stage in the energy expansion, ChPT is predictive only at sufficiently low energies, where the number of parameters introduced does not overwhelm the data to be described.

The prospects for extending ChPT in a useful way to calculations at finite density are unclear at present. On the other hand, the general framework of ChPT has validated the principle of resonance dominance of low-energy QCD. In particular, the $E^{4}$ coupling constants in $\mathrm{ChPT}$ in the meson sector are well reproduced from a meson resonance lagrangian applied at tree level, with the vector mesons playing the leading role [2]. Meson dominance is also the key principle underlying phenomenological models of nuclei with hadronic degrees of freedom, which we consider here. But while the correspondence in the vector channels is relatively straightforward because of well-defined resonances, the dynamics in the scalar channel is more difficult to identify and to model.

Within meson-exchange phenomenology, the mid-range attraction between nucleons is generally believed to be a dynamical consequence of the strong interactions between two pions exchanged with scalar, isoscalar quantum numbers [3]. No nearby underlying resonance at the relevant mass $(\approx 500 \mathrm{MeV})$ is evident or, in principle, needed. (Note that in ChPT investigations, the scalar resonance is identified with mesons around $1 \mathrm{GeV}$.) Nevertheless, this physics is efficiently, conveniently, and adequately represented at the one-meson exchange level by the exchange of a light scalar degree of freedom [4]. This light scalar is also an essential element of phenomenologically successful mean-field models of nuclei [5.6].

These mean-field models are significantly constrained by the bulk properties of finite nuclei [7 [9]. The question then arises: How should QCD symmetry constraints be manifested in these models? There is a long history of attempts to generalize the linear sigma model to build models with chiral symmetry; it is almost irresistible to identify the scalar meson mediating the mid-range nucleon-nucleon (NN) attraction with the chiral partner of the pion. More recently, interest in models realizing the broken scale invariance of QCD has been revived. Scale invariance is particularly compelling to consider because of its connection to the scalar channel. The breaking of scale invariance by the trace anomaly implies relations involving zero-momentum Green's functions of the scalar trace of the energy-momentum tensor [see Eqs. (2)-(3)]; these are called low-energy theorems [10]. If these relations are assumed to be saturated by scalar particles at tree level, significant constraints arise on the associated scalar potentials (in the chiral limit). We will exploit such constraints in this paper.

In Ref. [9], a broad class of models that attempt to unite successful mean-field phenomenology with chiral symmetry and the broken scale invariance of QCD were studied. Generalizations of the conventional linear sigma model that feature a "Mexican hat" potential were found to fail generically, even with modifications inspired by the realization of broken scale invariance. A significant improvement was found by the Minnesota group [11] when the "Mexican hat" potential is abandoned, and a reasonable description of the properties of closed-shell nuclei was obtained. 
In this paper, we build a different effective model of nuclei by implementing a nonlinear realization of chiral symmetry together with the low-energy theorems of broken scale invariance. The detailed construction of the full model will be reported elsewhere 112. Our focus here is primarily on how vacuum dynamics might be treated in an effective field theory of nuclei.

The role and manifestation of vacuum dynamics is an important issue in any fieldtheoretic description of nuclear matter and finite nuclei. Valence nucleons in the Fermi sea interact with each other and also with the QCD vacuum. In turn, the vacuum is modified by interactions with valence nucleons. In nonrelativistic models, such effects are never dealt with explicitly, but are absorbed implicitly into phenomenological effective interactions involving only valence nucleons. As a result, the interactions may acquire additional density dependence and nonlocalities. In previous relativistic models of nuclear matter involving a scalar field coupled to the nucleon, vacuum modifications were incorporated in the renormalized scalar effective potential [5,6]. This in turn affects the density dependence.

In principle, the one-baryon-loop effective potential contains an infinite number of undetermined coupling constants, which are the coefficients in a polynomial of infinite order in the scalar field. In conventional renormalizable models, the nucleon vacuum one-loop correction is well defined [13] and determines these coefficients, except for the terms of degree four and less, which are fixed by a renormalization prescription. However, renormalizable models with one-loop corrections do not achieve the phenomenological success of models without vacuum terms for the bulk properties of finite nuclei [14,15]. We interpret this failure as a phenomenological indication that the vacuum is not treated adequately.

In previous studies involving nonrenormalizable models, the effective potential is simply truncated, usually at degree four, and mean-field theory is applied without considering vacuum effects. In this paper, we begin to address the problem of constructing consistent calculations in effective field theories of nuclear matter and finite nuclei that explicitly address the role of the vacuum dynamics. In particular, we show how vacuum loop contributions are absorbed in the renormalization of coupling constants in the lagrangian in a model constrained to satisfy the low-energy theorems of QCD.

In contrast to the situation in ChPT, we cannot expand in powers of the energy, since we are not limited to derivative couplings and light meson masses. We observe, however, that the meson fields develop nonzero expectation values (mean fields) at finite density, and to begin, we assume that these mean fields dominate the contributions to the energy. Successful mean-field phenomenology shows that for densities not much higher than nuclear matter equilibrium density, the corresponding mean fields (or nucleon self-energies) are small compared to the free nucleon mass (roughly $\frac{1}{4}$ to $\frac{1}{3}$ the size); these ratios are therefore useful expansion parameters. Moreover, since the derivatives of the mean fields are small for normal nuclei, a truncation of the lagrangian at some low order of derivatives is also appropriate. (We verify this assertion explicitly later.) The end result is an energy functional for nuclear matter and nuclei that contains small powers of the mean fields and their derivatives; nuclear phenomenology implies that these fields are an efficient way to incorporate the density dependence of nuclear observables. Our objective here is to see how the low-energy behavior of QCD constrains the coefficients in this energy functional, particularly with regard to contributions from the quantum vacuum.

The assumption of mean-field dominance also has phenomenological support from Dirac- 
Brueckner-Hartree-Fock (DBHF) calculations, which indicate that exchange terms and short-range correlations do not significantly change the size of the nucleon self-energies nor introduce strong momentum dependence (at least for occupied states) [16, [7, [1]. Thus we have the favorable situation that the mean fields are large enough (compared to nuclear energy scales) to dominate the bulk dynamics but small enough (compared to the nucleon mass) to provide useful expansion parameters. Going beyond one-loop order systematically is an essential issue, but we will leave this as a topic for future study.

A nonlinear realization of chiral symmetry will be adopted, in which the Goldstone bosons (pions) are derivatively coupled to the nucleons. Historically, a linear representation (as in the usual linear sigma model) has been favored by model builders, in part because the sigma model is renormalizable. In this work, we wish to introduce a light scalar degree of freedom, but we do not want to make the restrictive dynamical assumption that this scalar is the chiral partner of the pions in a linear representation. By realizing chiral symmetry nonlinearly, we are not committed to such assumptions about the scalar degree of freedom. In addition, it will be easier to introduce vector mesons in a chirally invariant way that manifests the vector-meson dominance of Sakurai [18]. Finally, the nonlinear representation is more efficient for preserving the consequences of chiral symmetry at finite density when making approximations involving pions, because sensitive cancellations are not needed [6]. [7]

As suggested above, the nonderivative terms of the light scalar effective potential can be constrained by the low-energy theorems of QCD, so that vacuum effects are "built in." This, together with the truncation of our expansion in derivatives and powers of fields, leaves us with relatively few parameters, which can be determined by fitting to the properties of finite nuclei.

The paper is organized as follows: In Section II, broken scale invariance is discussed and the model is introduced and its renormalization is considered. An approximation scheme for nuclear matter and finite nuclei is proposed in Section III and the energy functional is derived. Results are given in Section IV. Section V contains some discussion of the results and Section VI is a summary.

\section{THE MODEL}

In meson-exchange phenomenology there is a light scalar degree of freedom that simulates two-pion-exchange physics in the scalar channel [3,.20]. Here we seek to describe this physics by introducing a light scalar field $S(x)$. We do not associate the scalar with a bound state or resonance, so we allow $S(x)$ to have anomalous behavior under a scale transformation in the effective theory. In particular, when $x \rightarrow \lambda^{-1} x, S(x) \rightarrow \lambda^{d} S(\lambda x)$, where $d$ can differ from unity and is to be determined phenomenologically. A QCD-inspired scenario that leads to such a scalar was proposed by Miransky and collaborators [21]. They introduced a light scalar generated by dynamical chiral symmetry breaking in $\mathrm{QCD}$, which was consequently

\footnotetext{
${ }^{1}$ We are unaware, however, of any proof of the independence of finite-density observables with respect to nonlinear field transformations, analogous to the theorem that applies to $S$-matrix elements [19].
} 
associated with the quark condensate $\langle\bar{q} q\rangle$ and referred to as quarkonium. We will take all other fields to have canonical scale dimension.

While massless QCD is scale invariant at the classical level, this symmetry is broken at the quantum level. This breaking is manifested in a nonzero trace of the energy-momentum tensor of QCD, which is referred to as the trace anomaly. The QCD trace anomaly in the chiral limit [22] is given by

$$
\theta_{\mu}^{\mu}(x) \equiv-H(x)=(\beta(g) / 2 g) G_{\mu \nu}^{a} G^{a \mu \nu}, \quad a=1,2, \cdots, N_{\mathrm{c}}^{2}-1,
$$

where $G_{\mu \nu}^{a}$ is the gluon field tensor and $\beta(g)=-\left(g^{3} / 48 \pi^{2}\right)\left(11 N_{\mathrm{c}}-2 N_{\mathrm{f}}\right)$ is the one-loop beta function with $N_{\mathrm{c}}$ colors and $N_{\mathrm{f}}$ flavors. There are remnants of scale invariance, which imply low-energy theorems that relate Green's functions involving the trace of the energymomentum tensor $H(x)$ [10]:

$$
\begin{aligned}
i \int \mathrm{d}^{4} x\langle 0|T[H(x) H(0)]| 0\rangle & =4 H_{0}, \\
i^{2} \int \mathrm{d}^{4} x \mathrm{~d}^{4} y\langle 0|T[H(x) H(y) H(0)]| 0\rangle & =4^{2} H_{0},
\end{aligned}
$$

where $H_{0} \equiv\langle 0|H| 0\rangle$.

Effective lagrangians for pure-glue QCD (no quarks) featuring a scalar glueball field $\chi(x)$ ("gluonium") that saturates these low-energy theorems at tree level have been considered many times [23]. Lattice QCD calculations indicate that the scalar glueball is quite heavy on hadronic mass scales, with a mass of roughly $1.6-1.8 \mathrm{GeV}$ [24]. Its fate in the real world with light quarks is not entirely clear. Here we will generalize the effective gluonium model to include the light scalar discussed earlier; this extension was proposed in a different context in Ref. [21]. We take the trace anomaly to consist of two contributions, corresponding to a vacuum expectation value $H_{0}=H_{\mathrm{g}}+H_{\mathrm{q}}$. Here $H_{\mathrm{g}}$ is identified with the heavy glueball contribution, while $H_{\mathrm{q}}$ is nonzero only when chiral symmetry is dynamically broken in the presence of light quarks. One can argue that $H_{\mathrm{g}}$ dominates $H_{0}$ (which is equal to the gluon condensate up to a factor) so that $H_{\mathrm{q}} \ll H_{\mathrm{g}}$ [21]. How the QCD trace anomaly actually separates into the two parts is not explored here, since we will determine $H_{\mathrm{q}}$ by fitting to the properties of finite nuclei. Nevertheless, we find that the value of $H_{\mathrm{q}}$ determined in our fits satisfies $H_{\mathrm{q}} \ll H_{0}$ (see Table @).

The low-energy theorems involving the trace $\theta_{\mu}^{\mu}(x)$ of the energy-momentum tensor are assumed to be saturated by the scalar gluonium $\chi(x)$ [23 and the light scalar $S(x)$. For simplicity we adopt a model with no mixing between the scalars. A candidate effective lagrangian of the scalars that satisfies the low-energy theorems at tree level in the chiral limit is 23,21

$$
\mathcal{L}_{\mathrm{s}}(x)=\frac{1}{2} \partial_{\mu} \chi \partial^{\mu} \chi+\frac{1}{2}\left[\alpha_{1}\left(\frac{\chi^{2}}{\chi_{0}^{2}}\right)^{1-d}+\left(1-\alpha_{1}\right)\left(\frac{S^{2}}{S_{0}^{2}}\right)^{(1-d) / d}\right] \partial_{\mu} S \partial^{\mu} S-V(\chi, S),
$$

where $\alpha_{1}$ is a real constant, $d$ is the scale dimension of the $S(x)$ field, and the scale-breaking potential $V$ is 


$$
V(\chi, S)=H_{\mathrm{g}} \frac{\chi^{4}}{\chi_{0}^{4}}\left(\ln \frac{\chi}{\chi_{0}}-\frac{1}{4}\right)+H_{\mathrm{q}}\left(\frac{S^{2}}{S_{0}^{2}}\right)^{2 / d}\left(\frac{1}{2 d} \ln \frac{S^{2}}{S_{0}^{2}}-\frac{1}{4}\right) .
$$

Here $\chi_{0}$ and $S_{0}$ are the vacuum expectation values of $\chi$ and $S$ respectively. Notice that $\alpha_{1}$ has been introduced so that after expanding the terms in square brackets in Eq. (4), the kinetic term for $S$ is canonical. The mass of the light scalar $S$ is given by $m_{\mathrm{s}}^{2}=4 H_{\mathrm{q}} /\left(d^{2} S_{0}^{2}\right)$. The scale dimension of the $\chi$ field is assumed to be unity.

One can define the energy-momentum tensor so that the Noether current for scale transformations is $x_{\nu} \theta^{\mu \nu}$. The trace of this "improved" energy-momentum tensor [25] corresponding to the lagrangian in Eqs. (4) and (5) is

$$
\begin{aligned}
\theta_{\mu}^{\mu}(x) & =S d \frac{\partial V}{\partial S}+\chi \frac{\partial V}{\partial \chi}-4 V(\chi, S) \\
& =-H_{\mathrm{g}} \frac{\chi^{4}}{\chi_{0}^{4}}-H_{\mathrm{q}}\left(\frac{S^{2}}{S_{0}^{2}}\right)^{2 / d} .
\end{aligned}
$$

With the dynamics of the scalar field fluctuations governed by the lagrangian in Eqs. (4) and (5), the preceding trace satisfies the low-energy theorems at the tree level. The usual direct demonstration [23], in which the gluonium alone is assumed to saturate the Green's functions, involves parametrizing the fluctuations $\tilde{\chi}(x)$ in the exponential form $\chi=\chi_{0} \exp \left[\widetilde{\chi}(x) / \chi_{0}\right]$ and substituting this into the low-energy theorems and into the potential, $V(\chi, 0)$ of Eq. (5), to determine vertices. Keeping only tree level diagrams (no loops), the theorems then follow.

To extend the demonstration to the present case, one first notes that the low-energy theorems should not depend on how the gluonium field fluctuation $\tilde{\chi}$ is parametrized. One then observes that if $\tilde{\chi}$ is defined through $\chi \equiv \chi_{0}\left(1-\tilde{\chi} / \chi_{0}\right)^{d}$, the resulting form for the gluonium parts of the trace and the potential in Eqs. (5) and (6) become the same as for the light scalar, when the fluctuation of the latter is parametrized simply as $S=S_{0}-\phi$. Since there are no couplings between the $\chi$ and the $S$ fields, the low-energy theorems follow directly.

We can now add to $\mathcal{L}_{s}$ a scale-invariant lagrangian with these scalars coupled to pion, nucleon, and vector degrees of freedom. (We neglect pion mass terms at this point.) The resulting model would be a candidate model for nuclei that satisfies the low-energy theorems. On the other hand, there are many other possible terms allowed, and even Eq. (田) is not the most general form involving two scalars. We choose to take advantage of the heaviness of the gluonium and the usefulness of an expansion in powers and derivatives of the other fields to both simplify and generalize the effective lagrangian. We expect the expansion to be valid and useful when applied near normal nuclear matter densities.

Since the mass scale of the heavy gluonium field (roughly $1.6-1.8 \mathrm{GeV}$ ) is significantly higher than the scales involved in the nuclear matter problem, the heavy gluonium field fluctuations $\tilde{\chi}$ can be integrated out as in Ref. [2]. In particular, we can eliminate $\tilde{\chi}$ by iteratively solving its equation of motion, exploiting the dominance of the mass term over powers and derivatives of $\tilde{\chi}$. This results in complicated terms involving powers and derivatives of the other fields, but we can expand these terms. For example, the second term in Eq. (州) would become

$$
\frac{1}{2}\left[1+\beta_{1} \frac{\phi}{S_{0}}+\beta_{2} \frac{\phi^{2}}{S_{0}^{2}}+\cdots\right] \partial_{\mu} \phi \partial^{\mu} \phi+\beta_{3}\left(\partial_{\mu} \phi \partial^{\mu} \phi\right)^{2}+\cdots,
$$


where the $\beta_{i}$ are functions of the constants $\chi_{0}, \alpha_{1}$, and $d$. The lagrangian will be much simpler if we can truncate this expansion at leading order in derivatives and neglect high powers of the meson fields.

We can follow this prescription to write a general chirally invariant effective lagrangian for nuclear matter and nuclei. The ground states of even-even nuclei and nuclear matter will be assumed to have good parity, so there is no pion mean field. Thus the pion will not play an explicit role in the present discussion of uniform nuclear matter and closed-shell nuclei in the Hartree approximation. Nevertheless, we wish to stress the connection to pion physics and the underlying constraints of chiral symmetry. Thus, we give an overview of the full model in order to motivate the form of the lagrangian and to set the stage for future work.

We restrict consideration to a low-energy representation of massless, two-flavor QCD. The Goldstone pion fields are represented by a chiral phase angle that corresponds to a pure chiral rotation of the identity matrix:

$$
\xi \mathbf{1} \xi \equiv U(x)=\exp \left(i \boldsymbol{\pi}(x) \cdot \boldsymbol{\tau} / f_{\pi}\right),
$$

where $\xi(x)=\exp \left(i \boldsymbol{\pi}(x) \cdot \boldsymbol{\tau} / 2 f_{\pi}\right), \tau^{a}(a=1,2$, and 3$)$ are the Pauli matrices, $\pi^{a}(x)$ are the Goldstone pion fields, and $f_{\pi}=93 \mathrm{MeV}$ is the pion-decay constant. This parametrization and the nucleon representation that follows is conventional; see, for example, Ref. [26]. The nucleon field is written as

$$
N(x)=\left(\begin{array}{c}
p(x) \\
n(x)
\end{array}\right),
$$

with $p(x)$ and $n(x)$ being the proton and neutron fields.

Under chiral transformations of $S U(2)_{\mathrm{L}} \otimes S U(2)_{\mathrm{R}}, U(x)$ transforms globally, $U(x) \rightarrow$ $L U(x) R^{\dagger}$, where $L=\exp \left(i \boldsymbol{\theta}_{\boldsymbol{L}} \cdot \boldsymbol{\tau}\right)$ and $R=\exp \left(i \boldsymbol{\theta}_{\boldsymbol{R}} \cdot \boldsymbol{\tau}\right)$ are $x$-independent elements of $S U(2)_{\mathrm{L}}$ and $S U(2)_{\mathrm{R}}$, respectively. In general, the transformation of $\xi$ is local since it depends on the pion field [28,26,27]:

$$
\xi(x) \rightarrow \xi^{\prime}(x)=L \xi(x) h^{\dagger}(x)=h(x) \xi(x) R^{\dagger},
$$

where the second equation defines the $S U(2)$-valued function $h(x)$ as a nonlinear function of $L, R$, and $U(x)$. Note that $h=L$ when $L=R$, i.e., in the case of a pure isospin rotation. The nucleon field also transforms locally: $N(x) \rightarrow h(x) N(x)$, which implies that nucleons mix with pions under chiral transformations. (See Ref. 28] for alternative representations of the nucleon field.)

We will incorporate the physics of vector dominance in our lagrangian by introducing vector mesons as gauge bosons [19]. For simplicity, since we concentrate on the properties of nearly symmetric $(N \approx Z)$ nuclear matter in this paper, we will not explicitly write down the rho and the electromagnetic fields. Thus only the $\omega$ meson field $V^{\mu}$ appears explicitly here. We will present a full discussion of the lagrangian elsewhere [12, including how the vector mesons are gauged and how vector dominance results. To build chirally invariant terms, it is useful to define the vector and axial vector fields

$$
\begin{aligned}
& v_{\mu}(x)=-\frac{i}{2}\left(\xi^{\dagger} \partial_{\mu} \xi+\xi \partial_{\mu} \xi^{\dagger}\right), \\
& a_{\mu}(x)=-\frac{i}{2}\left(\xi^{\dagger} \partial_{\mu} \xi-\xi \partial_{\mu} \xi^{\dagger}\right),
\end{aligned}
$$


which transform as $v_{\mu} \rightarrow h v_{\mu} h^{\dagger}-i h \partial_{\mu} h^{\dagger}$ and $a_{\mu} \rightarrow h a_{\mu} h^{\dagger}$. The coupling of the pion to the nucleon is realized through $a_{\mu}$ and the covariant derivative

$$
\mathcal{D}_{\mu}=\partial_{\mu}+i v_{\mu}+i g_{\mathrm{v}} V_{\mu}
$$

Now we can write the complete chirally invariant lagrangian; all terms not contained in $\mathcal{L}_{s}$ are scale invariant. After integrating out $\tilde{\chi}$ and expanding about $S_{0}$, the lagrangian takes the form

$$
\begin{aligned}
\mathcal{L}(x)= & \bar{N}\left(i \gamma^{\mu} \mathcal{D}_{\mu}-i g_{\mathrm{A}} \gamma^{\mu} \gamma_{5} a_{\mu}-M+g_{\mathrm{s}} \phi+\cdots\right) N-\frac{1}{4} F_{\mu \nu} F^{\mu \nu} \\
& +\frac{1}{2}\left[1+\eta \frac{\phi}{S_{0}}+\cdots\right]\left[\frac{1}{2} f_{\pi}^{2} \operatorname{tr}\left(\partial_{\mu} U \partial^{\mu} U^{\dagger}\right)+m_{\mathrm{v}}^{2} V_{\mu} V^{\mu}\right] \\
& +\frac{1}{4 !} \zeta\left(g_{\mathrm{v}}^{2} V_{\mu} V^{\mu}\right)^{2}+\frac{1}{2} \partial_{\mu} \phi \partial^{\mu} \phi-H_{\mathrm{q}}\left(\frac{S^{2}}{S_{0}^{2}}\right)^{2 / d}\left(\frac{1}{2 d} \ln \frac{S^{2}}{S_{0}^{2}}-\frac{1}{4}\right)+\cdots
\end{aligned}
$$

where $g_{\mathrm{A}}=1.23$ is the axial coupling constant, $g_{\mathrm{s}}\left(g_{\mathrm{v}}\right)$ is the light scalar (vector $\omega$ ) coupling to the nucleon, the $\omega$ field strength tensor is $F_{\mu \nu}=\partial_{\mu} V_{\nu}-\partial_{\nu} V_{\mu}$, and $\eta$ and $\zeta$ are real constants.

Several features of this lagrangian are of interest:

- We have combined terms after expanding and have rewritten the coefficients, where appropriate, in terms of physical masses. Note that the nucleon mass $M$ has contributions from the vacuum expectation values of both scalars; we do not assume that it comes entirely from the light scalar (although this possibility is not excluded).

- The combination of the $\omega$ mass term and the pion kinetic term in Eq. (14) appears naturally, if we assume the vector mesons to be gauge bosons [19, 12.

- The original separation of the lagrangian into a scale-invariant piece and a scalebreaking piece, in which the latter involved only the scalar fields, is now largely hidden because the $\chi$ dependence is not explicit and we have expanded about $S_{0}$. Nevertheless, there is a remnant for our purposes here: the scale-breaking potential of the light scalar [the last term in Eq. (14)], which is not changed by the elimination of $\tilde{\chi}$. (Recall that the $\chi$ and $S$ do not mix.) Thus the low-energy theorems still protect the form of this potential, which places constraints on vacuum loop renormalizations, as discussed below.

- We have omitted many higher-order terms, as indicated by the ellipses, which represent higher powers of fields and their derivatives. Only Yukawa couplings to the nucleon fields are kept, based on the phenomenological dominance of one-meson exchange and the implicit elimination of heavier fields. (So $\bar{N} N \phi^{2}$ terms, etc., are omitted.) Higherorder terms with meson fields should give numerically small contributions (in nuclei) or can be absorbed into slight adjustments of the other parameters. Some explicit justification for these claims is given in the results below.

The lagrangian in Eq. (14) is written with renormalized coefficients. Counterterms are not written explicitly, but are implied. In particular, these counterterms include all powers 
of the scalar field, not just terms up to $O\left(\phi^{4}\right)$, as in a renormalizable model. To understand how these counterterms are fixed, we start by integrating out the baryon fields at zero density and temperature. The result is a fermion determinant that contributes to the meson action as an additive term given by

$$
S_{\mathrm{fd}}\left[\phi, V_{\mu}\right] \equiv \int \mathrm{d}^{4} x \mathcal{L}_{\mathrm{fd}}=-i \operatorname{Tr} \ln K(0),
$$

where "Tr" indicates a trace over spacetime, spin, and isospin, and the kernel $K(\mu)$ is defined in coordinate space by

$$
\langle x|K(\mu)| y\rangle=\left[i \gamma^{\mu} \partial_{\mu}-g_{\mathrm{v}} \gamma^{\mu} V_{\mu}(x)+\mu \gamma_{0}-M+g_{\mathrm{s}} \phi(x)\right] \delta^{4}(x-y) .
$$

The introduction of the chemical potential $\mu$ is for later convenience, and baryon counterterms, which are needed beyond one-loop, are suppressed. Note that no approximation has been made at this point; $S_{\mathrm{fd}}$ is a functional of the dynamical fields $\phi$ and $V^{\mu}$ that still must be integrated over in a path integral, for example. The techniques for expanding a determinant in powers of derivatives can be found in Ref. [29]; see also the heat-kernel method in Ref. [26]. The expansion of Eq. (15) in a renormalizable model has been discussed in Ref. 30].

We first focus on the nonderivative terms, which can be obtained from Eq. (15) by treating the fields as constants and by expanding the logarithm in a power series in the fields. Baryon number conservation implies that for the vector field, only its derivatives can appear in the expansion. Thus the nonderivative part of $\mathcal{L}_{\mathrm{fd}}$ is an infinite polynomial in $\phi$; for example, at the one-loop level,

$$
\mathcal{L}_{\mathrm{fd}}[\phi]=i \int \frac{\mathrm{d}^{\tau} k}{(2 \pi)^{4}} \operatorname{tr} \ln G^{0}(k)+i \sum_{n=1}^{\infty} \frac{(-1)^{n}}{n}\left[g_{\mathrm{s}} \phi(x)\right]^{n} \int \frac{\mathrm{d}^{\tau} k}{(2 \pi)^{4}} \operatorname{tr}\left[G^{0}(k)\right]^{n} .
$$

Here we have regularized dimensionally to maintain Lorentz covariance and baryon number conservation, "tr" denotes a trace over spin and isospin only, and

$$
G^{0}(k)=\frac{1}{\not k-M+i \epsilon}
$$

is the free baryon propagator. Beyond one loop there are additional terms in the coefficients, including baryon counterterm contributions.

The polynomial in $\phi$ of Eq. (17) must be combined with the corresponding counterterms; in this way the vacuum contributions are absorbed into the renormalization of the scalar polynomial. If one insists that the low-energy theorems be satisfied at tree level in the meson fields, the end result for the scalar potential should be of the form in Eq. (14), where the couplings are renormalized. (Note that this potential can be expanded as a polynomial in $\phi$, with all coefficients determined by $H_{q}, S_{0}$, and $d$.) One never has to explicitly calculate any counterterms or evaluate Eq. (17); when we write down the scalar potential, the nucleon-loop effects have already been taken into account. Furthermore, although we have illustrated the renormalization by evaluating nucleon loops only, any additional baryonic degrees of freedom in the lagrangian would be treated analogously and the final result will be the same. Thus the phenomenological fitting of parameters accommodates a general characterization of the vacuum response. 
The renormalization of the derivative terms is analogous except that we do not have low-energy theorems to reduce the number of renormalized coupling constants. We note, however, that each additional derivative is accompanied by an inverse power of a typical scale in the problem, which is the nucleon mass here. Experience with mean-field models of nuclei also suggest that the derivatives of the mean fields are small (for example, $|\nabla \phi / \phi| \lesssim 100 \mathrm{MeV})$. Thus if we assume mean-field dominance, such that fluctuations around the mean fields are small, and the naturalness of the coefficients in the derivative expansion (see the discussion in Section V), we can truncate the derivative terms at some tractable order. In this work, we will stop at the lowest order for the derivatives. Thus we have only a few unknown renormalized constants (parameters), which are determined by fitting to experiment; in our case, we will use finite-density observables.

At finite density, we work in the grand canonical ensemble through the introduction of a chemical potential $\mu$ [31]. We consider only zero temperature in this work, which allows a simplified discussion. The relevant lagrangian density is now

$$
\mathcal{L}^{\prime}(x, \mu)=\mathcal{L}(x)+\mu \bar{N} \gamma_{0} N .
$$

Here the effective action of $\mathcal{L}^{\prime}$ is associated with the thermodynamic potential $\Omega$ of the system, instead of the energy. The energy follows from

$$
E=\Omega+\mu B,
$$

where

$$
B=-\frac{\partial \Omega}{\partial \mu}
$$

is the baryon number of the system.

Now we integrate out the baryon field as at zero density. The result is the fermion determinant at finite density (or chemical potential), $-i \operatorname{Tr} \ln K(\mu)$, to which we can add and subtract the fermion determinant at $\mu=0,-i \operatorname{Tr} \ln K(0)$. [ The added term $-i \operatorname{Tr} \ln K(0)$ combines with the counterterms exactly as described above so that the renormalization goes through as before. Note that it contains the same dynamical scalar and vector fields as the fermion determinant at $\mu$. The remaining combination

$$
-i \operatorname{Tr} \ln K(\mu)+i \operatorname{Tr} \ln K(0)
$$

is an explicitly density-dependent piece (it vanishes for $\mu=0$ ), which is finite if baryon counterterms are included in $K(\mu)$. (This combination is evaluated in the Hartree approximation in the next section, for which the baryon counterterms are not needed.) Once again the scalar potential in the form shown in Eq. (14) is left intact; the only difference is that the scalar field now acquires a different expectation value due to the presence of valence nucleons at finite density.

\footnotetext{
${ }^{2}$ We assume that $\mu=0$ still separates the positive-energy levels from the Dirac sea. This will be the case if the density is not too high.
} 


\section{FINITE NUCLEI AND NUCLEAR MATTER}

To perform a realistic calculation, we need a good starting approximation. Since our focus here is on bulk nuclear properties and on single-particle spectra, we assume that the mean meson fields dominate the dynamics, and we expand the finite-density thermodynamic potential around the mean fields. The lowest-order result (Hartree approximation) is obtained by replacing all the meson fields by their mean values, and this will be the starting point of any systematic approximation for treating the fluctuations.

The thermodynamic potential for nuclei in the Hartree approximation is given by

$$
\int \mathrm{d} x_{0} \Omega=i \operatorname{Tr} \ln \bar{K}(\mu)-i \operatorname{Tr} \ln \bar{K}(0)-\int \mathrm{d}^{4} x U_{\mathrm{m}}(\mathbf{x}),
$$

where the baryon kernel in coordinate space is now

$$
\langle x|\bar{K}(\mu)| y\rangle=\gamma_{0}\left[i \partial_{0}+\mu-h(\mathbf{x})\right] \delta^{(4)}(x-y) .
$$

The single particle hamiltonian $h$ is

$$
h(\mathbf{x})=-i \boldsymbol{\alpha} \cdot \boldsymbol{\nabla}+g_{\mathrm{v}} V_{0}(\mathbf{x})+\beta\left(M-g_{\mathrm{s}} \phi_{0}(\mathbf{x})\right),
$$

with $\beta=\gamma_{0}$ and $\boldsymbol{\alpha}=\gamma_{0} \boldsymbol{\gamma}$, and the static scalar and vector mean fields are denoted by $\phi_{0}(\mathbf{x})$ and $V_{0}(\mathbf{x})$. The contribution from the meson fields is

$$
\begin{aligned}
U_{\mathrm{m}}(\mathbf{x})= & -\frac{1}{2}\left(\boldsymbol{\nabla} \phi_{0}\right)^{2}-\frac{1}{4} m_{\mathrm{s}}^{2} S_{0}^{2} d^{2}\left\{\left(1-\frac{\phi_{0}}{S_{0}}\right)^{4 / d}\left[\frac{1}{d} \ln \left(1-\frac{\phi_{0}}{S_{0}}\right)-\frac{1}{4}\right]+\frac{1}{4}\right\} \\
& +\frac{1}{2}\left(\nabla V_{0}\right)^{2}+\frac{1}{2}\left(1+\eta \frac{\phi_{0}}{S_{0}}\right) m_{\mathrm{v}}^{2} V_{0}^{2}+\frac{1}{4 !} \zeta\left(g_{\mathrm{v}} V_{0}\right)^{4} .
\end{aligned}
$$

Note that $\bar{K}(\mu)$ is diagonal in the single-particle basis $\psi_{\alpha}(\mathbf{x}) e^{i \omega x_{0}}$, where $\psi_{\alpha}(\mathbf{x})$ are the normalized eigenfunctions of the Dirac equation with eigenvalues $E_{\alpha}$ [32,6]:

$$
h \psi_{\alpha}(\mathbf{x})=E_{\alpha} \psi_{\alpha}(\mathbf{x}), \quad \int \mathrm{d}^{3} x \psi_{\alpha}^{\dagger}(\mathbf{x}) \psi_{\alpha}(\mathbf{x})=1
$$

From a path integral formulation, one can see that the appropriate boundary condition or $i \epsilon$ prescription for evaluating the baryon kernel is $\omega \rightarrow(1+i \epsilon) \omega$.

From Eq. (23) one can now obtain, after a Wick rotation,

$$
\begin{aligned}
\Omega & =-\sum_{\alpha} \int \frac{\mathrm{d} \omega}{2 \pi}\left[\ln \left(-i \omega+\mu-E_{\alpha}\right)-\ln \left(-i \omega-E_{\alpha}\right)\right]-\int \mathrm{d}^{3} x U_{\mathrm{m}} \\
& =-\sum_{\alpha}\left(\mu-E_{\alpha}\right)\left[\theta\left(\mu-E_{\alpha}\right)-\theta\left(-E_{\alpha}\right)\right]-\int \mathrm{d}^{3} x U_{\mathrm{m}} \\
& \equiv-\sum_{\alpha}^{\text {occ }}\left(\mu-E_{\alpha}\right)-\int \mathrm{d}^{3} x U_{\mathrm{m}} .
\end{aligned}
$$

Here we have used

$$
\sum_{\alpha} \theta\left(-E_{\alpha}\right)=\sum_{\alpha} \theta\left(E_{\alpha}\right)=\sum_{\alpha} \frac{1}{2}
$$


which is valid when $\mu=0$ separates the nucleon levels from the antinucleon levels. The summation superscript "occ" means that the sum runs only over occupied states in the Fermi sea. Moreover, using Eqs. (20) and (21), we find

$$
\begin{aligned}
& B=\sum_{\alpha}\left[\theta\left(\mu-E_{\alpha}\right)-\theta\left(-E_{\alpha}\right)\right]=\sum_{\alpha}^{\mathrm{occ}} 1, \\
& E=\sum_{\alpha}^{\mathrm{occ}} E_{\alpha}-\int \mathrm{d}^{3} x U_{\mathrm{m}} .
\end{aligned}
$$

We emphasize that the final sum over only occupied (valence) states is not the result of a vacuum subtraction, as the term with $\mu=0$ still contains the background fields, which must be determined self-consistently. The true vacuum subtraction was performed earlier when we derived the renormalized $U_{\mathrm{m}}$.

The equations for the mean fields are obtained from extremizing the energy functional with respect to $\phi_{0}(\mathbf{x})$ and $V_{0}(\mathbf{x})$. From Eqs. (25) and (27) one finds

$$
\begin{aligned}
\frac{\delta E_{\alpha}}{\delta \phi_{0}(\mathbf{x})} & =\frac{\delta}{\delta \phi_{0}(\mathbf{x})} \int \mathrm{d}^{3} y \psi_{\alpha}^{\dagger}(\mathbf{y}) h(\mathbf{y}) \psi_{\alpha}(\mathbf{y}) \\
& =\psi_{\alpha}^{\dagger}(\mathbf{x}) \frac{\partial h}{\partial \phi_{0}} \psi_{\alpha}(\mathbf{x})+E_{\alpha} \frac{\delta}{\delta \phi_{0}(\mathbf{x})} \int \mathrm{d}^{3} y \psi_{\alpha}^{\dagger}(\mathbf{y}) \psi_{\alpha}(\mathbf{y}) \\
& =\psi_{\alpha}^{\dagger}(\mathbf{x}) \frac{\partial h}{\partial \phi_{0}} \psi_{\alpha}(\mathbf{x})
\end{aligned}
$$

and a similar expression for the variation with respect to $V_{0}$; evaluating the derivatives yields

$$
\begin{aligned}
& \frac{\delta}{\delta \phi_{0}(\mathbf{x})} \sum_{\alpha}^{\mathrm{occ}} E_{\alpha}=-g_{\mathrm{s}} \sum_{\alpha}^{\mathrm{occ}} \bar{\psi}_{\alpha}(\mathbf{x}) \psi_{\alpha}(\mathbf{x}) \\
& \frac{\delta}{\delta V_{0}(\mathbf{x})} \sum_{\alpha}^{\mathrm{occ}} E_{\alpha}=g_{\mathrm{v}} \sum_{\alpha}^{\mathrm{occ}} \psi_{\alpha}^{\dagger}(\mathbf{x}) \psi_{\alpha}(\mathbf{x})
\end{aligned}
$$

Upon applying these results to Eq. (31), one obtains the mean-field equations:

$$
\begin{aligned}
-\nabla^{2} \phi_{0}+m_{\mathrm{s}}^{2} \phi_{0}= & g_{\mathrm{s}} \sum_{\alpha}^{\mathrm{occ}} \bar{\psi}_{\alpha}(\mathbf{x}) \psi_{\alpha}(\mathbf{x}) \\
& +m_{\mathrm{s}}^{2} \phi_{0}+m_{\mathrm{s}}^{2} S_{0}\left(1-\frac{\phi_{0}}{S_{0}}\right)^{(4 / d)-1} \ln \left(1-\frac{\phi_{0}}{S_{0}}\right)+\frac{\eta}{2 S_{0}} m_{\mathrm{v}}^{2} V_{0}^{2}, \\
-\nabla^{2} V_{0}+m_{\mathrm{v}}^{2} V_{0}= & g_{\mathrm{v}} \sum_{\alpha}^{\text {occ }} \psi_{\alpha}^{\dagger}(\mathbf{x}) \psi_{\alpha}(\mathbf{x}) \\
& -\eta \frac{\phi_{0}}{S_{0}} m_{\mathrm{v}}^{2} V_{0}-\frac{1}{6} \zeta g_{\mathrm{v}}^{4} V_{0}^{3} .
\end{aligned}
$$

Note that we have added an explicit mass term to each side of the scalar field equation to put it in a form that can be solved with conventional numerical techniques [32]. The vector mean-field equation is actually a constraint since the time component of the vector field is not a dynamical degree of freedom. (See below for further comments in the case of nuclear matter.) 
This lowest-order result (Hartree approximation) is similar to that obtained from conventional derivations of relativistic mean-field models in which one-loop vacuum corrections are simply neglected. We emphasize, however, that we are not merely presenting another meanfield model; the vacuum effects are incorporated and systematic improvement is possible (in principle). Rather, we consider our procedure a justification for the phenomenologically successful mean-field approach.

The energy density for uniform nuclear matter in the Hartree approximation can be obtained from the preceding results by observing that the single-particle energy eigenvalue becomes

$$
E(\mathbf{k})=g_{\mathrm{v}} V_{0}+\sqrt{\mathbf{k}^{2}+M^{* 2}},
$$

where $M^{*}=M-g_{\mathrm{s}} \phi_{0}$, and $\phi_{0}$ and $V_{0}$ are now constant mean fields. The energy density $\mathcal{E}$ becomes

$$
\begin{aligned}
\mathcal{E}\left[M^{*}, \rho_{\mathrm{B}}\right]= & \frac{1}{4} m_{\mathrm{s}}^{2} S_{0}^{2} d^{2}\left\{\left(1-\frac{\phi_{0}}{S_{0}}\right)^{4 / d}\left[\frac{1}{d} \ln \left(1-\frac{\phi_{0}}{S_{0}}\right)-\frac{1}{4}\right]+\frac{1}{4}\right\}+g_{\mathrm{v}} \rho_{\mathrm{B}} V_{0}-\frac{1}{4 !} \zeta\left(g_{\mathrm{v}} V_{0}\right)^{4} \\
& -\frac{1}{2}\left(1+\eta \frac{\phi_{0}}{S_{0}}\right) m_{\mathrm{v}}^{2} V_{0}^{2}+\frac{\gamma}{(2 \pi)^{3}} \int^{k_{\mathrm{F}}} \mathrm{d}^{3} k \sqrt{\mathbf{k}^{2}+M^{* 2}}
\end{aligned}
$$

where $k_{\mathrm{F}}$ is the Fermi momentum defined by $\mu=g_{\mathrm{v}} V_{0}+\sqrt{k_{\mathrm{F}}^{2}+M^{* 2}}$, and $\rho_{\mathrm{B}}=\gamma k_{\mathrm{F}}^{3} /\left(6 \pi^{2}\right)$ is the baryon density. The spin-isospin degeneracy $\gamma=4$ for nuclear matter and $\gamma=2$ for neutron matter.

The equation that determines $V_{0}$ can be obtained either from the Euler-Lagrange equations or by using Dirac's procedure [33], with the result

$$
g_{\mathrm{v}} \rho_{\mathrm{B}}=\left(1+\eta \frac{\phi_{0}}{S_{0}}\right) m_{\mathrm{v}}^{2} V_{0}+\frac{1}{6} \zeta g_{\mathrm{v}}^{4} V_{0}^{3} .
$$

This equation can also be obtained from Eq. (38) by setting $\left(\partial \mathcal{E} / \partial V_{0}\right)_{\rho_{\mathrm{B}}, M^{*}}=0$. Note, however, that this is not a minimization condition for $\mathcal{E}$. In fact, the $V_{0}$ obtained from Eq. (39) corresponds to a local maximum of the energy density. Equation (39), like Eq. (36), is a constraint equation for $V_{0}$, which is not a dynamical variable.

The energy density at a given baryon density is found by using Eq. (39) to eliminate $V_{0}$ from $\mathcal{E}$ in Eq. (38) and then by minimizing the resulting finite-density effective potential with respect to $M^{*}$. The effective potential at fixed baryon density is shown in Fig. 1. Notice that in contrast to the conventional one-loop approximation (relativistic Hartree approximation or RHA [5,6]) in renormalizable models, the finite-density effective potential of our truncated model is meaningful only when $\left|g_{\mathrm{s}} \phi_{0}\right|$ is sufficiently small that higher-order terms can be neglected. Similar considerations apply to the solutions of Eq. (39). (See Section V for further discussion.) Parameters can be chosen so that nuclear matter exhibits saturation at the empirical point; one approach to determining the parameters is discussed in the next section.

\section{RESULTS}

To test the utility of the model, we must see if it can successfully describe finite nuclei [9]. The basic features we seek to reproduce are the nuclear charge densities (including 


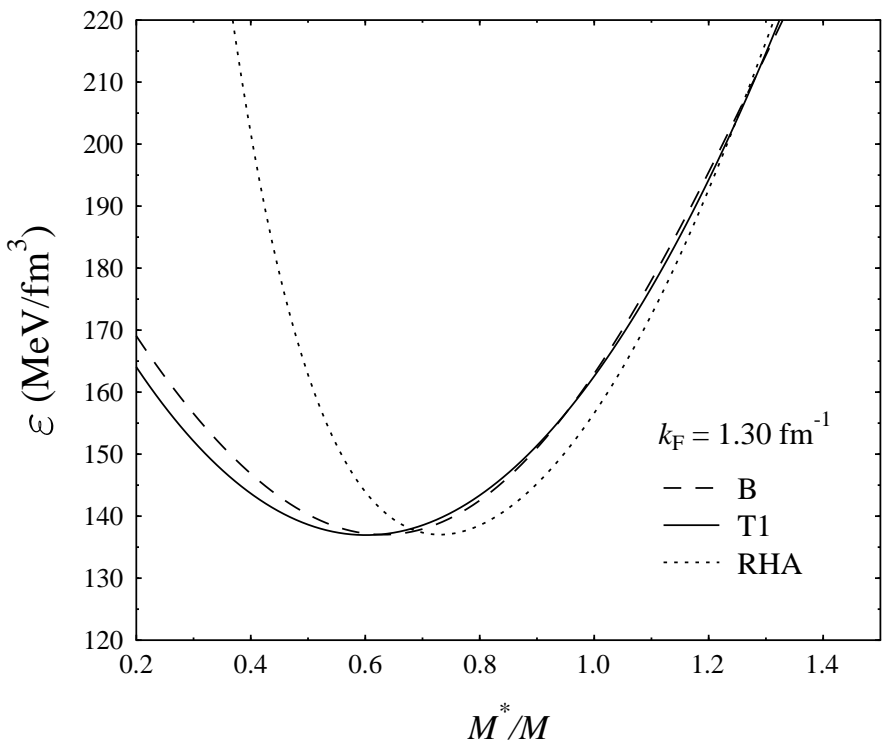

FIG. 1. Finite-density effective potential $\mathcal{E}$ from Eq. (38), plotted as a function of $M^{*}$ (solid line). $V_{0}$ is eliminated for each $M^{*}$ using Eq. (39). Parameter set T1 is used and $k_{\mathrm{F}}=1.30 \mathrm{fm}^{-1}$. Results for other parameter sets and other densities are qualitatively similar. Also shown are the analogous potentials for the Walecka model RHA [5] (dotted line) and the nonlinear parameter set B from Ref. [9] (dashed line).

the observed flatness in heavy nuclei), the characteristics of the single-particle spectrum, and the bulk binding-energy systematics. Relativistic mean-field models unconstrained by QCD symmetries have been successful in reproducing these properties for nuclei across the periodic table.

The Hartree equations for finite nuclei in our model were given in Section III, but only isoscalar mesons were discussed. To make realistic comparisons to experiment, we must include the $\rho$ and the Coulomb interactions. Here we simply introduce the $\rho$ and the photon as in Ref. 9], except that we also include a coupling between the $\rho$ and the scalar $\phi$, exactly as for the $\omega$ [see Eq. (38)]. A more complete treatment of the isovector mesons will be presented elsewhere [12].

We take the nucleon, $\omega$, and $\rho$ masses as given by their experimental values: $M=$ $939 \mathrm{MeV}, m_{\mathrm{v}}=783 \mathrm{MeV}$, and $m_{\rho}=770 \mathrm{MeV}$. We then fit the rest of the parameters $\left(g_{\mathrm{s}}\right.$, $g_{\mathrm{v}}, g_{\rho}, \eta, \zeta, m_{\mathrm{s}}, S_{0}$, and $\left.d\right)$ to the binding energies, the charge radii, and the spin-orbit splittings of the least-bound proton and neutron in ${ }^{16} \mathrm{O},{ }^{40} \mathrm{Ca}$, and ${ }^{208} \mathrm{~Pb}$, as well as to the charge density of ${ }^{16} \mathrm{O}$ at $r \approx 1 \mathrm{fm}$. An optimization process similar to that of Ref. [35] is used. Here we are principally interested in showing that a good fit to properties of finite

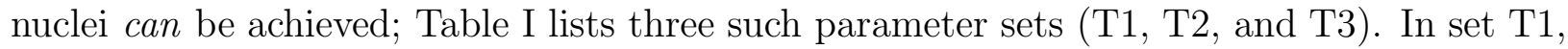
$d$ is an optimization parameter, while it is fixed (arbitrarily) in sets T2 and T3 to illustrate the range of possible $d$. In a future paper, we will study in more detail the regions of the parameter space that produce a reasonable fit and examine which conditions are important in determining individual parameters.

We have calculated ${ }^{16} \mathrm{O},{ }^{40} \mathrm{Ca}$, and ${ }^{208} \mathrm{~Pb}$ for these parameter sets and for a representative 
TABLE I. Parameter sets from fits to finite nuclei. The vector masses are $m_{\mathrm{v}}=783 \mathrm{MeV}$ and $m_{\rho}=770 \mathrm{MeV}$; the nucleon mass is $M=939 \mathrm{MeV}$. Values for $S_{0}$, the scalar mass $m_{\mathrm{s}}$, and $H_{\mathrm{q}}^{1 / 4}$ are in $\mathrm{MeV}$. Note that $m_{\mathrm{s}}^{2}=4 H_{\mathrm{q}} /\left(d^{2} S_{0}^{2}\right)$.

\begin{tabular}{lccccccccc}
\hline \hline Set & $g_{\mathrm{s}}^{2}$ & $m_{\mathrm{s}}$ & $g_{\mathrm{v}}^{2}$ & $g_{\rho}^{2}$ & $S_{0}$ & $\zeta$ & $\eta$ & $d$ & $H_{\mathrm{q}}^{1 / 4}$ \\
\hline T1 & 99.3 & 509. & 154.5 & 70.2 & 90.6 & 0.0402 & -0.496 & 2.70 & 250. \\
T2 & 96.3 & 529. & 138.0 & 69.6 & 95.6 & 0.0342 & -0.701 & 2.20 & 236. \\
T3 & 109.5 & 508. & 178.6 & 67.2 & 89.8 & 0.0346 & -0.160 & 3.50 & 283. \\
\hline \hline
\end{tabular}

TABLE II. Binding-energy systematics for the model proposed here (sets T1, T2, and T3), for model B from Ref. 9], and for the point-coupling (PC) model of Ref. [35. Binding energies per nucleon are given in $\mathrm{MeV}$.

\begin{tabular}{cccc}
\hline \hline Model & ${ }^{16} \mathrm{O}$ & ${ }^{40} \mathrm{Ca}$ & ${ }^{208} \mathrm{~Pb}$ \\
\hline T1 & 7.99 & 8.61 & 7.91 \\
T2 & 7.94 & 8.55 & 7.89 \\
T3 & 7.95 & 8.53 & 7.91 \\
B & 7.82 & 8.35 & 7.62 \\
PC & 7.97 & 8.58 & 7.87 \\
exp't & 7.98 & 8.55 & 7.87 \\
\hline \hline
\end{tabular}

mean-field model (set B from Ref. [9]). Bulk binding-energy systematics are summarized in Table $\mathbb{1 1}$ and rms charge radii are summarized in Table 111 . For comparison, we also include results from the point-coupling model of Ref. [35]. The binding energies include center-ofmass corrections as in Ref. [7]. We show charge densities and single-particle levels for ${ }^{208} \mathrm{~Pb}$ in Figs. 2 and 3, and charge densities for ${ }^{16} \mathrm{O}$ and ${ }^{40} \mathrm{Ca}$ in Figs. 1 and 5. The charge densities are determined from point-proton densities following the conventional procedure [32], which folds them with a phenomenological proton form factor. Form factors generated within the model itself, originating from vector dominance physics, will be considered elsewhere.

The fits to nuclear charge radii, binding energies, and spin-orbit splittings are quite good. The only deficiencies in the sets illustrated here are some small deviations from experiment in the charge densities. Changes in the optimization procedure can improve the agreement of the charge densities at the cost of worsening slightly the agreement with empirical binding

TABLE III. Rms charge radii (in fm) for the model proposed here (sets T1, T2, and T3), for model B from Ref. [9], and for the point-coupling (PC) model of Ref. [35].

\begin{tabular}{cccc}
\hline \hline Model & ${ }^{16} \mathrm{O}$ & ${ }^{40} \mathrm{Ca}$ & ${ }^{208} \mathrm{~Pb}$ \\
\hline T1 & 2.73 & 3.47 & 5.56 \\
T2 & 2.72 & 3.47 & 5.56 \\
T3 & 2.72 & 3.48 & 5.57 \\
B & 2.74 & 3.48 & 5.56 \\
PC & 2.73 & 3.45 & 5.51 \\
exp't & 2.74 & 3.47 & 5.50 \\
\hline \hline
\end{tabular}




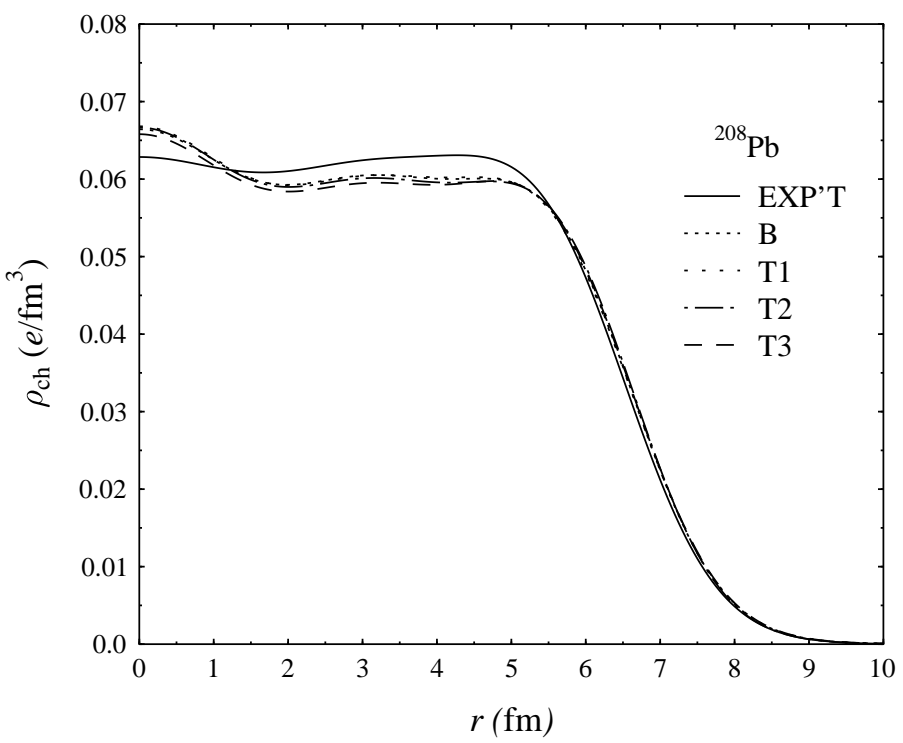

FIG. 2. Charge density of ${ }^{208} \mathrm{~Pb}$. The solid line is taken from experiment [37]. Charge densities are shown for a successful mean-field model (model B from Ref. [9]) and for the three parameter sets from Table I.

energies.

A good reproduction of the spin-orbit force in finite nuclei necessarily leads to large scalar and vector mean fields in the interiors of the nuclei or in nuclear matter. In particular, as discussed many times (recently by Bodmer [8]), vector and scalar fields of roughly 250$300 \mathrm{MeV}$ are needed to reproduce the observed spin-orbit splittings in the least-bound levels (and also the deformations in light, axially symmetric nuclei [15]). While these fields are large on the scale of the nuclear binding energies, $\left|g_{\mathrm{v}} V_{0}\right| / M$ and $\left|g_{\mathrm{s}} \phi_{0}\right| / M$ and their gradients in finite nuclei are relatively small; thus, these remain useful expansion parameters. This justifies our truncation of the energy density at small powers of the meson fields. While it is possible in principle to add additional monomials in the fields (with undetermined parameters), the quality of the present fit makes it unlikely that there is much to be gained by this.

The scale dimension $d$ of the light scalar field was found to be about 2.7 when $d$ was included in the optimization. Note that the canonical dimension would have $d=1$. Changes in the optimization procedure or a relaxation in the goals of the fit allow for a considerable range in $d$ (sets $\mathrm{T} 2$ and $\mathrm{T} 3$ are examples), but it does not seem possible to find a reasonable parameter set with $d<2$. Thus the introduction of an anomalous dimension for the light scalar degree of freedom is an essential feature for the phenomenological success of our model.

Experience with a broad class of relativistic mean-field models shows that models that successfully reproduce bulk properties of finite nuclei share characteristic properties in infinite nuclear matter [9]. These properties are the equilibrium binding energy and density, the compressibility $K$, and the value of $M^{*} / M$ at equilibrium. One further condition, that the light scalar mass $m_{\mathrm{s}} \approx 500 \mathrm{MeV}$, is needed to ensure reasonably smooth charge densities and good surface-energy systematics. If we calculate nuclear matter with the parameter sets 


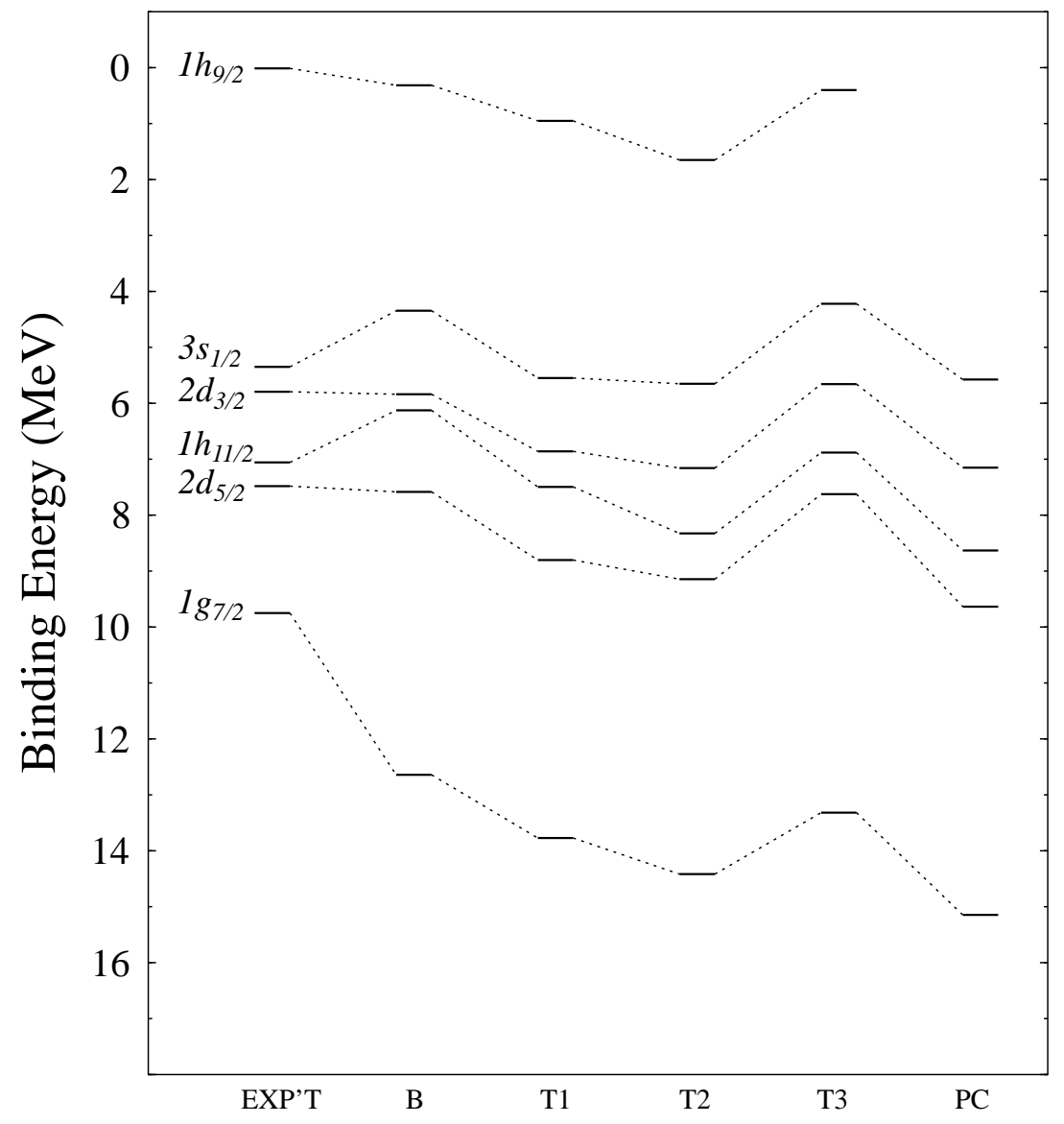

FIG. 3. Predicted proton single-particle spectra for ${ }^{208} \mathrm{~Pb}$ using the parameter sets from Table I. Only the least-bound major shell is shown. The leftmost values are from experiment, model B is a successful mean-field model from Ref. [9], and model PC is the point-coupling model of Ref. [35]. Note that the $1 h_{9 / 2}$ level is an unoccupied state. 


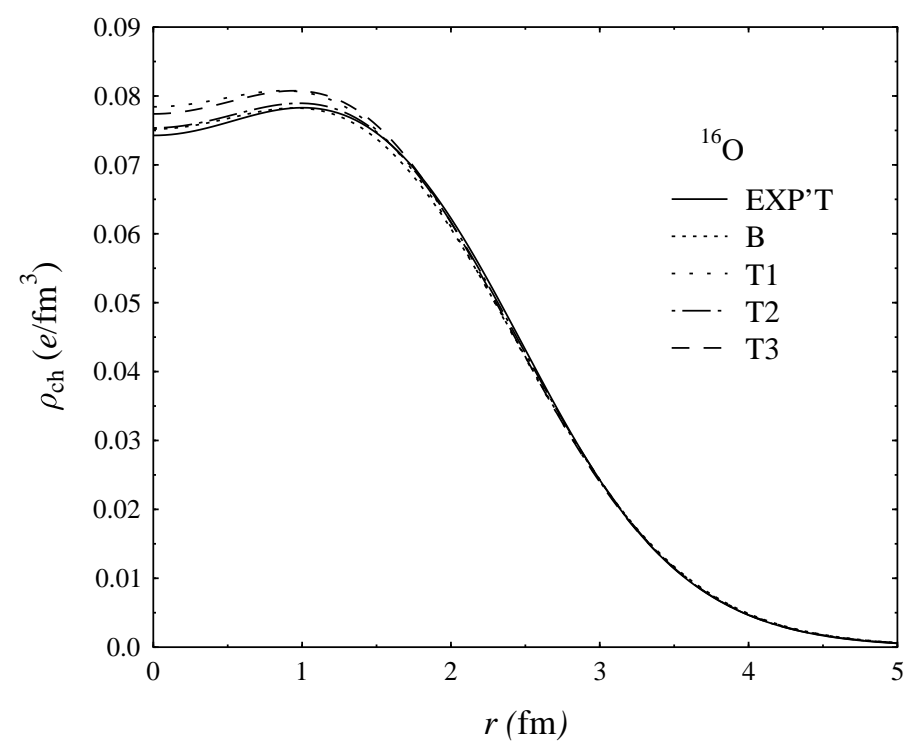

FIG. 4. Charge density of ${ }^{16} \mathrm{O}$. The solid line is taken from experiment 37]. Charge densities are shown for a successful mean-field model (model B from Ref. [9]) and for the three parameter sets from Table I.

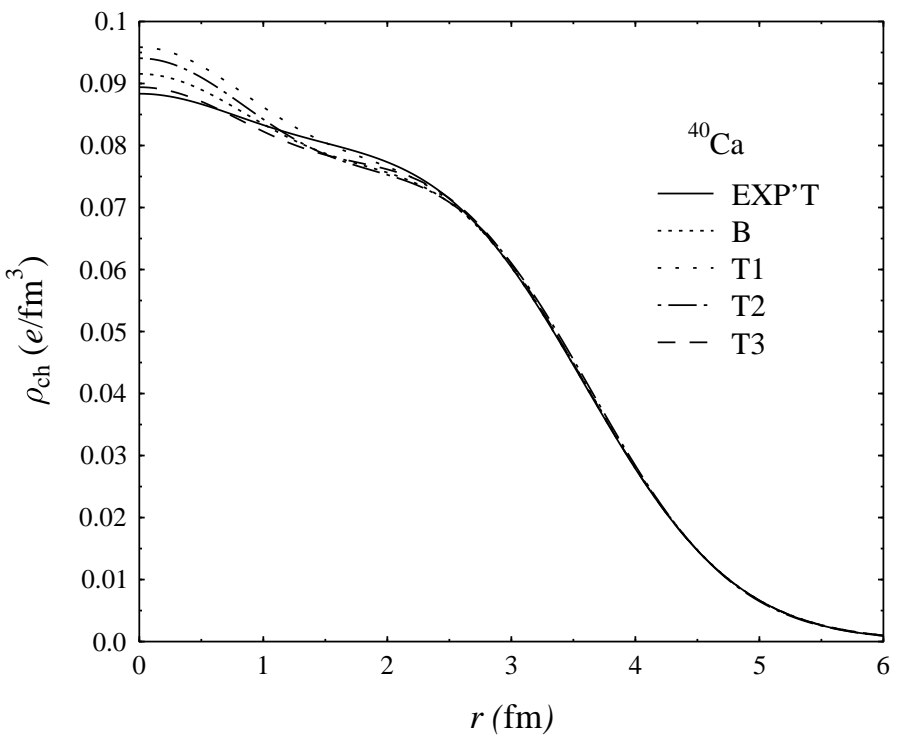

FIG. 5. Charge density of ${ }^{40} \mathrm{Ca}$. The solid line is taken from experiment 37]. Charge densities are shown for a successful mean-field model (model B from Ref. [9]) and for the three parameter sets from Table I. 
TABLE IV. Nuclear matter saturation properties for the model proposed here (sets T1, T2, and T3), for model B from Ref. [9], and for the point-coupling (PC) model of Ref. [35]. Values are given for the binding energy per nucleon (in $\mathrm{MeV}$ ), the Fermi momentum $k_{\mathrm{F}}\left(\mathrm{in} \mathrm{fm}^{-1}\right.$ ), the compressibility $K$ (in $\mathrm{MeV}$ ), the bulk symmetry energy coefficient $a_{4}$ (in $\mathrm{MeV}$ ), $M^{*} / M$, and $g_{\mathrm{v}} V_{0}$ (in $\mathrm{MeV}$ ) at equilibrium.

\begin{tabular}{ccccccc}
\hline \hline Model & $E / B-M$ & $k_{\mathrm{F}}$ & $K$ & $a_{4}$ & $M^{*} / M$ & $g_{\mathrm{v}} V_{0}$ \\
\hline T1 & 16.2 & 1.30 & 194. & 39. & 0.60 & 302. \\
T2 & 16.3 & 1.29 & 240. & 40. & 0.61 & 298. \\
T3 & 16.1 & 1.29 & 244. & 34. & 0.61 & 297. \\
B & 15.8 & 1.30 & 220. & 35. & 0.63 & 277. \\
PC & 16.1 & 1.30 & 264. & & 0.58 & 322. \\
\hline \hline
\end{tabular}

in Table 1, we find good agreement with values found in investigations with unconstrained mean-field models (see Table IV). In particular, the saturation density corresponds to a Fermi momentum of about $1.3 \mathrm{fm}^{-1}$, and the binding energy per nucleon at saturation is about $16 \mathrm{MeV}$. The compressibility is less well determined (190-250 MeV). The nucleon effective mass $M^{*} / M \approx 0.60$ and the scalar mass $m_{\mathrm{s}}$ is just over $500 \mathrm{MeV}$. We emphasize that these values are obtained after a fit to finite nuclei only.

\section{DISCUSSION}

We can relate the phenomenological success of the model proposed here to the characteristics of successful relativistic mean-field models of finite nuclei. A key feature is the logarithmic potential for the scalar field, which allows for relatively weak nonlinearities and the dominance of the cubic and quartic scalar terms, with the values of the scaling dimension $d$ used here. In contrast, chiral models with a Mexican hat potential have large cubic and quartic terms, which preclude a good fit to bulk nuclear properties [9]. Bodmer [8] has shown that nuclear matter properties that lead to good predictions for finite nuclei can be achieved if one adds to (small) cubic and quartic scalar terms a term that is quartic in the vector field (here with coupling $\zeta$ ). Thus our model has all of the ingredients needed to allow a good fit through optimization. In addition, adjustments can be made through the scalar-vector coupling $\eta$.

Note that the scalar-vector coupling and the quartic vector self-coupling can be used to define an effective, density-dependent mass of the vector meson at the mean-field level. For example, one can use the second derivative of the lagrangian with respect to the vector field. For the model parameters in Table [ 1 , the two contributions largely cancel, so that the vector effective mass $m_{\mathrm{v}}^{*}$ is essentially independent of density. This is in contrast to the universal scaling hypothesis of Brown and Rho [36], which predicts $m_{\mathrm{v}}^{*} / m_{\mathrm{v}}=M^{*} / M$.

We have excluded many terms from our model: higher-order polynomials in the vector fields and mixed scalar-vector terms, non-Yukawa couplings to the nucleon, derivative terms, and so on. In retrospect, were we justified in neglecting them? An analysis of mean-field models [8,12] implies that one can identify dimensionless ratios that can be used to set the scale of individual contributions to the energy. For example, one can rewrite the scaled 
energy density of nuclear matter, $\mathcal{E} / M^{4}$, in terms of the dimensionless ratios $g_{\mathrm{v}} V_{0} / M$ and $g_{\mathrm{s}} \phi_{0} / M$, which then become our finite-density expansion parameters.

Moreover, an important assumption in applying effective field theories, such as chiral perturbation theory, is that the coefficients of terms in the lagrangian are "natural," i.e., of order unity, when written in appropriate dimensionless units. This assumption makes the organization of terms through a power-counting scheme useful, because one can systematically truncate the expansion when working to a desired accuracy. We have proposed an analogous concept of naturalness for the finite-density problem, which will justify the neglect of higher derivatives and powers of the fields when applying Eq. (14) to nuclei. For example, if one expresses the nuclear matter energy density in terms of the scaled field variables written above, one finds that the ratios

$$
\frac{m_{\mathrm{v}}^{2}}{2 g_{\mathrm{v}}^{2} M^{2}}, \quad \frac{m_{\mathrm{s}}^{2}}{2 g_{\mathrm{s}}^{2} M^{2}}, \quad \frac{\zeta}{8}, \frac{\eta m_{\mathrm{v}}^{2}}{2 g_{\mathrm{s}} g_{\mathrm{v}}^{2} S_{0} M},
$$

should all be of roughly equal size for our expansion to be "natural." One can verify that the values in sets $\mathrm{T} 1, \mathrm{~T} 2$, and T3 satisfy this condition.

To examine the size of the scalar self-interactions, one expands the logarithmic potential in Eq. (38) with the result

$$
\begin{aligned}
\frac{1}{4} m_{\mathrm{s}}^{2} S_{0}^{2} d^{2}\{(1- & \left.\left.\frac{\phi_{0}}{S_{0}}\right)^{4 / d}\left[\frac{1}{d} \ln \left(1-\frac{\phi_{0}}{S_{0}}\right)-\frac{1}{4}\right]+\frac{1}{4}\right\} / M^{4} \\
= & {\left[\frac{m_{\mathrm{s}}^{2}}{2 ! g_{\mathrm{s}}^{2} M^{2}}\right] \widetilde{\Phi}^{2}+\left[\frac{1}{3 ! M g_{\mathrm{s}}^{3}} \frac{(3 d-8) m_{\mathrm{s}}^{2}}{d S_{0}}\right] \widetilde{\Phi}^{3}+\left[\frac{1}{4 ! g_{\mathrm{s}}^{4}} \frac{\left(11 d^{2}-48 d+48\right) m_{\mathrm{s}}^{2}}{\left(d S_{0}\right)^{2}}\right] \widetilde{\Phi}^{4} } \\
& +\left[\frac{M}{5 ! g_{\mathrm{s}}^{5}} \frac{2\left(25 d^{3}-140 d^{2}+240 d-128\right) m_{\mathrm{s}}^{2}}{\left(d S_{0}\right)^{3}}\right] \widetilde{\Phi}^{5} \\
& +\left[\frac{M^{2}}{6 ! g_{\mathrm{s}}^{6}} \frac{2\left(137 d^{4}-900 d^{3}+2040 d^{2}-1920 d+640\right) m_{\mathrm{s}}^{2}}{\left(d S_{0}\right)^{4}}\right] \widetilde{\Phi}^{6}+\cdots
\end{aligned}
$$

where $\widetilde{\Phi} \equiv g_{\mathrm{s}} \phi_{0} / M$. The coefficients in square brackets give the combinations that should be compared to those in Eq. (40), and one can verify that these are also natural for parameter sets T1, T2, and T3. It is interesting that for set T1, in which $d$ is an optimized parameter, the scaled coefficients are extremely small due to nearly complete cancellations among terms in the polynomials in $d$. (Further discussion of these issues is given in Ref. [12].)

Further support for the naturalness assumption comes from extending the model to include $\phi^{2} V_{\mu} V^{\mu}$ and $\left(V_{\mu} V^{\mu}\right)^{3}$ terms and then repeating the optimization. The new fit is very close to the fit obtained without these terms. Furthermore, contributions to the energy from the new terms are less than $10 \%$ of those from the old terms at nuclear matter density, and the old coefficients change only slightly in the new fit 12. Thus contributions from the higher-order terms can be absorbed into slight adjustments of the coefficients in Eq. (14).

The astute reader will note that if our naturalness assumption is justified, we could construct a variation of our model without the constraints of the low-energy theorems of broken scale invariance. Indeed, at nuclear matter density, numerics alone would let us truncate the scalar potential, and the same arguments about renormalization apply, so that vacuum effects are still built in. This explains the success of previous relativistic mean-field 
models of nuclear structure and illustrates the power of the assumption of naturalness. Here we note that the scalar potential constrained by the low-energy theorems actually provides some justification for naturalness, which we simply assume is valid for higher-order and derivative terms. Thus one should be cautious in drawing strong conclusions about the role of broken scale invariance when applied in models that are restricted to moderate nuclear density.

How widely can our model be applied? A prime motivation for developing relativistic models of nuclei and nuclear matter is to extrapolate to extremes of density and temperature [5]. Such conditions can be reached experimentally in relativistic heavy-ion collisions. One hopes that the calibration of such models to observables at ordinary nuclear densities and zero temperature, in conjunction with constraints from QCD symmetries, will permit reliable extrapolations.

Unfortunately, our framework of mean-field dominance, naturalness, and the truncation at small powers of the fields and their derivatives, which limits the number of parameters at ordinary nuclear densities, is bound to break down as the density increases. With increasing density, we will find increasing mean fields and expansion parameters that are no longer small. Thus we become increasingly less justified in ignoring the effects of higher-order terms, and the calibration at nuclear matter density becomes less and less of a constraint. The limits of reliable extrapolation are not clear, but one should certainly be cautious in applying models like ours much above nuclear matter density. Nevertheless, the utility of an accurate relativistic mean-field model for nuclear structure and reactions, which is compatible with the low-energy behavior of QCD, should be obvious.

We close our discussion with some interesting observations. From Table [I, one sees that $S_{0}$, the vacuum expectation value of the light scalar field $S$, is close to the experimental value of $f_{\pi}(93 \mathrm{MeV})$. Furthermore, the scalar coupling constant $g_{\mathrm{s}}$ is close to $g_{\pi} / g_{A}$. If we forget for the moment complications from requiring terms to be scale invariant, it is tempting to say that the model has a preference for the nucleon mass to be generated entirely from the vacuum expectation value of $S$. That is, if the only nucleon coupling to scalar fields is

$g_{\mathrm{s}} \bar{N} N S$, then we recover the empirical nucleon mass and the Goldberger-Treiman relation from the fit values of the other parameters. This scenario is also consistent with Miransky's model, in which the light scalar (quarkonium) is associated with the quark condensate, and with QCD sum rules, which associate the nucleon mass predominantly with the quark condensate. It is premature to do more than to point out these results, but the coincidence of numbers certainly merits further investigation.

\section{SUMMARY}

In summary, we have introduced a new model for nuclear matter and finite nuclei that realizes QCD symmetries at the hadronic level. In particular, the model incorporates chiral symmetry, broken scale invariance, and the phenomenology of vector dominance. An important feature is the light scalar degree of freedom, which is given an anomalous scale dimension. The renormalized scalar potential is constrained by the low-energy theorems of broken scale invariance. Vacuum loop effects are absorbed into the renormalized parameters, which are determined by fits to hadron masses and finite-density observables. 
The truncation of the model lagrangian is based on mean-field dominance and the identification of expansion parameters that are reasonably small at nuclear matter densities. Due to the characteristics of the constrained scalar potential, we adopt a "naturalness" assumption, which justifies the truncation. The parameters of the truncated model are identified by an optimization procedure designed to reproduce bulk properties of finite nuclei. Good fits are obtained, which also lead to very reasonable nuclear matter properties. The scale dimension of the scalar field comes out greater than two, but is not tightly constrained by the fit.

It is important to emphasize what we have learned about the relationship between effective (hadronic) theories of QCD and successful relativistic mean-field phenomenology. The vacuum dynamics of $\mathrm{QCD}$ is constrained by the trace anomaly and the consequent lowenergy theorems of QCD. At the level of hadronic fields, this physics manifests itself in the scalar-isoscalar sector of the theory. We have proposed that this sector can be divided into a low-mass part that is adequately described by a scalar meson with anomalous dimension and a high-mass part that is "integrated out," leading to various couplings among the remaining fields. We believe this latter characterization is quite general and independent of the details of the high-mass part of the scalar sector. Nevertheless, whereas the realization of the Goldstone boson dynamics is well known (i.e., chiral perturbation theory), as is the dynamics of the vector sector (i.e., vector-meson dominance), little is known about the precise form and magnitudes of the nonlinear couplings originating from the scalar degrees of freedom. We find that our primary source of information on this dynamics comes from nuclear structure physics, which provides strong constraints on this sector of the theory.

In subsequent work, we will further explore the parameter space that leads to good fits to nuclear properties and identify the observables that constrain individual terms. We will also investigate the chiral properties of the model and study the implications of vector dominance for nuclear observables. Work to extend the model beyond the one-baryon-loop level in a

manner consistent with conservation laws and Ward identities is in progress. Finally, we will continue the development of the naturalness concept for finite-density systems.

\section{ACKNOWLEDGMENTS}

We are pleased to thank P. J. Ellis, S. V. Gardner, C. J. Horowitz, D. B. Leinweber, V. A. Miransky, R. J. Perry, S. Rudaz, and J. Rusnak for useful comments. This work was supported in part by the National Science Foundation under Grant Nos. PHY-9203145, PHY-9258270, PHY-9207889, and PHY-9102922, and the Sloan Foundation and by the U.S. Department of Energy under contract No. DE-FG02-87ER40365. 


\section{REFERENCES}

[1] S. Weinberg, Phys. Rev. 166, 1568 (1968); Physica A 96, 327 (1979);

R. Dashen and M. Weinstein, Phys. Rev. 183, 1261(1969);

J. Gasser and H. Leutwyler, Ann. Phys. (N.Y.) 158, 142 (1984); Nucl. Phys. B250, 465, 517, 539 (1985).

[2] G. Ecker, J. Gasser, A. Pich, and E. De Rafael, Nucl. Phys. B321, 311 (1989);

G. Ecker, J. Gasser, H. Leutwyler, and E. De Rafael, Phys. Lett. B223, 425 (1989);

J. F. Donoghue, C. Ramirez, and G. Valencia, Phys. Rev. D 39, 1947 (1989).

[3] J. W. Durso, A. D. Jackson, and B. J. VerWest, Nucl. Phys. A345, 471 (1980).

[4] R. Machleidt, Adv. Nucl. Phys. 19, 189 (1989).

[5] J. D. Walecka, Ann. Phys. (N.Y.) 83, 491 (1974).

[6] B. D. Serot and J. D. Walecka, Adv. Nucl. Phys. 16, 1 (1986);

B. D. Serot, Rep. Prog. Phys. 55, 1855 (1992).

[7] P.-G. Reinhard, Rep. Prog. Phys. 52, 439 (1989).

[8] A. R. Bodmer, Nucl. Phys. A526, 703 (1991).

[9] R. J. Furnstahl and B. D. Serot, Phys. Rev. C 47, 2338 (1993); Phys. Lett. B 316, 12 (1993).

[10] A. I. Vainshtein, V. I. Zakharov, V. A. Novikov, and M. A. Shifman, Sov. J. Part. Nucl. 13, 224 (1982).

[11] E. K. Heide, S. Rudaz, and P. J. Ellis, Nucl. Phys. A571, 713 (1994).

[12] R. J. Furnstahl, H. B. Tang, and B. D. Serot, in preparation.

[13] S.A. Chin, Ann. Phys. (N.Y.) 108, 301 (1977).

[14] W. R. Fox, Nucl. Phys. A495, 463 (1989).

[15] R. J. Furnstahl, C. E. Price, and G. E. Walker, Phys. Rev. C 36, 2590 (1987).

[16] C. J. Horowitz and B. D. Serot, Nucl. Phys. A464, 613 (1987); A473, 760 (1987) (E).

[17] B. ter Haar and R. Malfliet, Phys. Rep. 149, 207 (1987).

[18] J. J. Sakurai, Currents and Mesons (University of Chicago Press, Chicago, IL, 1969).

[19] M. Bando, T. Kugo, and K. Yamawaki, Phys. Rep. 164, 217 (1988).

[20] W. Lin and B. D. Serot, Nucl. Phys. A512, 637 (1990).

[21] V. A. Miransky and V. P. Gusynin, Prog. Theor. Phys. 81, 426 (1989).

[22] J. Collins, A. Duncan, and S. D. Jogelkar, Phys. Rev. D 16, 438 (1977);

N. K. Nielson, Nucl. Phys. B120, 212 (1977);

D. G. Robertson, Phys. Lett. B 253, 143 (1991).

[23] J. Schechter, Phys. Rev. D 213393 (1980);

A. A. Migdal and M. A. Shifman, Phys. Lett. 114B, 445 (1982);

J. Ellis and J. Lanik, Phys. Lett. 150B, 289 (1985).

[24] D. Weingarten, Nucl. Phys. B (Proc. Suppl.) 34, 29 (1994).

[25] C. Callan, S. Coleman, and R. Jackiw, Ann. Phys. (N.Y.) 59, 42 (1970).

[26] J. F. Donoghue, E. Golowich, and B. R. Holstein, Dynamics of the Standard Model (Cambridge University Press, New York, 1992).

[27] C. Callan, S. Coleman, J. Wess, and B. Zumino, Phys. Rev. 177, 2247 (1969).

[28] H. Georgi, Weak Interaction and Modern Particle Theory (Benjamin/Cummings, Menlo Park, CA, 1984).

[29] I. J. R. Aitchison and C. M. Fraser, Phys. Lett. 146B, 63 (1984);

O. Cheyette, Phys. Rev. Lett. 55, 2394 (1985); 
C. M. Fraser, Z. Phys. C 28, 101 (1985);

L. H. Chan, Phys. Rev. Lett. 54, 1222 (1985).

[30] R. J. Perry, Phys. Lett. 182B, 269 (1986); R. J. Furnstahl and C. E. Price, Phys. Rev. C 41, 1792 (1990).

[31] H. B. Tang, Ph. D. thesis, Indiana University (1993), unpublished.

[32] C. J. Horowitz and B. D. Serot, Nucl. Phys. A368, 503 (1981).

[33] P. A. M. Dirac, Lectures on Quantum Mechanics, Belfer Grad. School of Science (Yeshiva University, New York, 1964).

[34] T. D. Lee and G. C. Wick, Phys. Rev. D 9, 2291 (1974).

[35] B. A. Nikolaus, T. Hoch, and D. G. Madland, Phys. Rev. C 46, 1757 (1992).

[36] G. E. Brown and M. Rho, Phys. Rev. Lett. 66, 2720 (1991).

[37] H. de Vries, C. W. de Jager, and C. de Vries, Atomic Data and Nuclear Data Tables 36, 495 (1987). 